\title{
Maximum Weight Triangulation and Graph Drawing*
}

\author{
Cao An Wang ${ }^{1}$, Francis Y. Chin ${ }^{2}$, and Bo Ting Yang ${ }^{1}$ \\ 1 Department of Computer Science, Memorial University of Newfoundland, \\ St. John's, Newfoundland, Canada A1B 3X5 \\ wang@garfield.cs.mun.ca \\ 2 Department of Computer Science, University of Hong Kong, Hong Kong \\ chin@cs.hku.hk
}

\section{Introduction}

Triangulation of a set of points is a fundamental structure in computational geometry. According to the authors' best knowledge, there is not much research done on maximum weight triangulation, MaxWT. From the theoretical viewpoint, MaxWT and its counterpart, the minimum weight triangulation, attract equally interest. The graph drawings as MaxWT are investigated.

- The weight of a triangulation $T(P)$ is given by

$$
\omega(T(P))=\sum_{\overline{p_{i} p_{j}} \in T(P)} \omega\left(\overline{p_{i} p_{j}}\right),
$$

where $\omega\left(\overline{p_{i} p_{j}}\right)$ is the Euclidean length of line segment $\overline{p_{i} p_{j}}$.

- A maximum weight triangulation of $P(\operatorname{Max} W T(P))$ is defined as for all possible $T(P), \omega(\operatorname{Max} W T(P))=\max \{\omega(T(P))\}$.

\section{Main Results}

(A) $\mathrm{O}\left(n^{2}\right)$ time algorithm for MaxWT of an inscribed $n$-gon.

(B) $\mathrm{O}(n)$ time algorithm for MaxWT of a regular $n$-gon.

(C) $\mathrm{O}\left(n^{2}\right)$ time 0.5 -approximation algorithm for MaxWT of a general convex $n$-gon.

(D) Linear-time algorithm for maximum drawing of Caterpillar graphs.

(E) Forbidden (non-maximum weight drawable) graphs on any convex point set.

\section{(A) Inscribed Polygon Case}

FACT 1: If $P$ is a convex polygon, then each interior angle of any fly triangle of the $\operatorname{Max} W T(P)$ must be no less than $\frac{\pi}{4}$.

FACT 2: If $P$ is a convex polygon, then no interior angle of any fly triangle of $\operatorname{Max} W T(P)$ is larger than $\frac{\pi}{2}$.

\footnotetext{
* This work is supported by NSERC grant OPG0041629 and RGC grant HKU $541 / 96 \mathrm{E}$.
} 
FACT 3: If $P$ is an inscribed polygon. Then $\operatorname{Max} W T(P)$ cannot contain any fly triangle. That is, the internal edges of $\operatorname{Max} W T(P)$ form a tree.

\section{Recurrence Formula}

- $W_{i, j}$ - The weight of convex subpolygon of the inscribed polygon $P$ with vertics $(i, i+1, \ldots, j)$ for $i, j \epsilon[0, n]$.

$$
W_{i, j}= \begin{cases}0 & \text { if } j=(i+1)_{\text {modulo. } n} \\ \max \left\{W_{i, j-1}, W_{i+1, j}\right\}+\omega(\overline{i j}) & \text { otherwise }\end{cases}
$$

- $\mathrm{O}\left(n^{2}\right)$ time algorithm.

\section{(B) Regular Polygon Case}

- Any inner-spanning tree of a regular $n$-gon $P$ is maximum and it together with the boundary edges of $P$ form a $\operatorname{Max} W T(P)$.

- An inner-spanning tree of a regular $n$-gon $P$ can be found in linear time.

\section{(C) A 0.5-Approximation Algorithm for $\operatorname{MaxWT}(\mathrm{P})$}

- Let $\triangle a b c$ be a fly triangle of $\operatorname{Max} W T(P)$. The removal of a fly triangle $\triangle a b c$ will divide $P$ into three components, each associates with an edge of $\triangle a b c$. $\triangle a b c$ is called an ear-fly triangle if at most one of its three components contains other fly triangles.

- An $\left(n^{2}\right)$ time approximate algorithm which guarantees $\frac{\omega(A p T(P))}{\omega(\operatorname{Max} W T(P))} \geq \frac{1}{2}$.

\section{(D) Maximum Weight Drawing of Caterpillar Graph}

- caterpillar is a tree such that all internal nodes connect to at most 2 non-leaf nodes.

- $\mathrm{O}(n)$ time algorithm.

\section{(E) Forbidden Graphs for Maximum Weight Drawing}

- A graph $G$ is outerplanar if it has a planar embedding such that all its nodes lie on a single face; an outerplanar graph is maximal if no edge can be added to the planar embedding without crossing.

- If $G(V, E)$ is a maximal outerplanar graph containing a simple cycle $C$ with four nonconsecutive nodes which form two triangles sharing a common edge, then $G$ cannot have a maximum weight drawing.

\section{Concluding Remarks}

- Does MaxWT(P) can be found in o $\left(n^{3}\right)$ time?

- Does every maxmal planar graph admit a maximum weight drawing? 